\title{
Integral method coefficients and regularization procedure for the ring- core residual stress measurement technique
}

\section{Michele Barsanti $^{1, \mathrm{a}}$, Marco Beghini ${ }^{1, \mathrm{~b}}$, Ciro Santus $^{1, \mathrm{c}^{*}}$, Alessio Benincasa ${ }^{2, \mathrm{~d}}$ and Lorenzo Bertelli ${ }^{2, e}$}

\footnotetext{
${ }^{1}$ University of Pisa - Dipartimento di Ingegneria Civile e Industriale, DICI. Largo L. Lazzarino 2, 56122 Pisa. Italy.
}

${ }^{2}$ SINT Technology Srl. Via delle Calandre 63, 50041 Calenzano, Florence. Italy.

am.barsanti@ing.unipi.it, bm.beghini@ing.unipi.it, cciro.santus@ing.unipi.it,

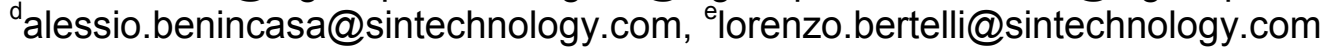

Keywords: Ring-core method; Integral method; Tikhonov regularization; FE modeling.

\begin{abstract}
The ring-core method allows the determination of residual stresses at high depth from the surface. The numerical calculation integral method, commonly used for the hole-drilling, can also be applied to the ring-core. The integral method coefficients were obtained for several depth steps after axial-symmetric FE simulations with harmonic load. These coefficients were then validated with a 3D finite element model. Finally, an application was reported, showing the performance of the Tikhonov regularization on experimental data.
\end{abstract}

\section{Introduction}

Hole-drilling and ring-core are both mechanical methods based on axial-symmetric material removal. The material is cut at the center and the relieved strain measured at the periphery for the hole-drilling, while the opposite for the ring-core. The hole-drilling is more widespread being dedicated to superficial stresses [1-6]. The ring-core, introduced many years ago [7,8], has diameters and also depth much larger (Fig. 1 shows the dimension comparison) so this technique is usually dedicated to large size structures $[9,10,11]$.
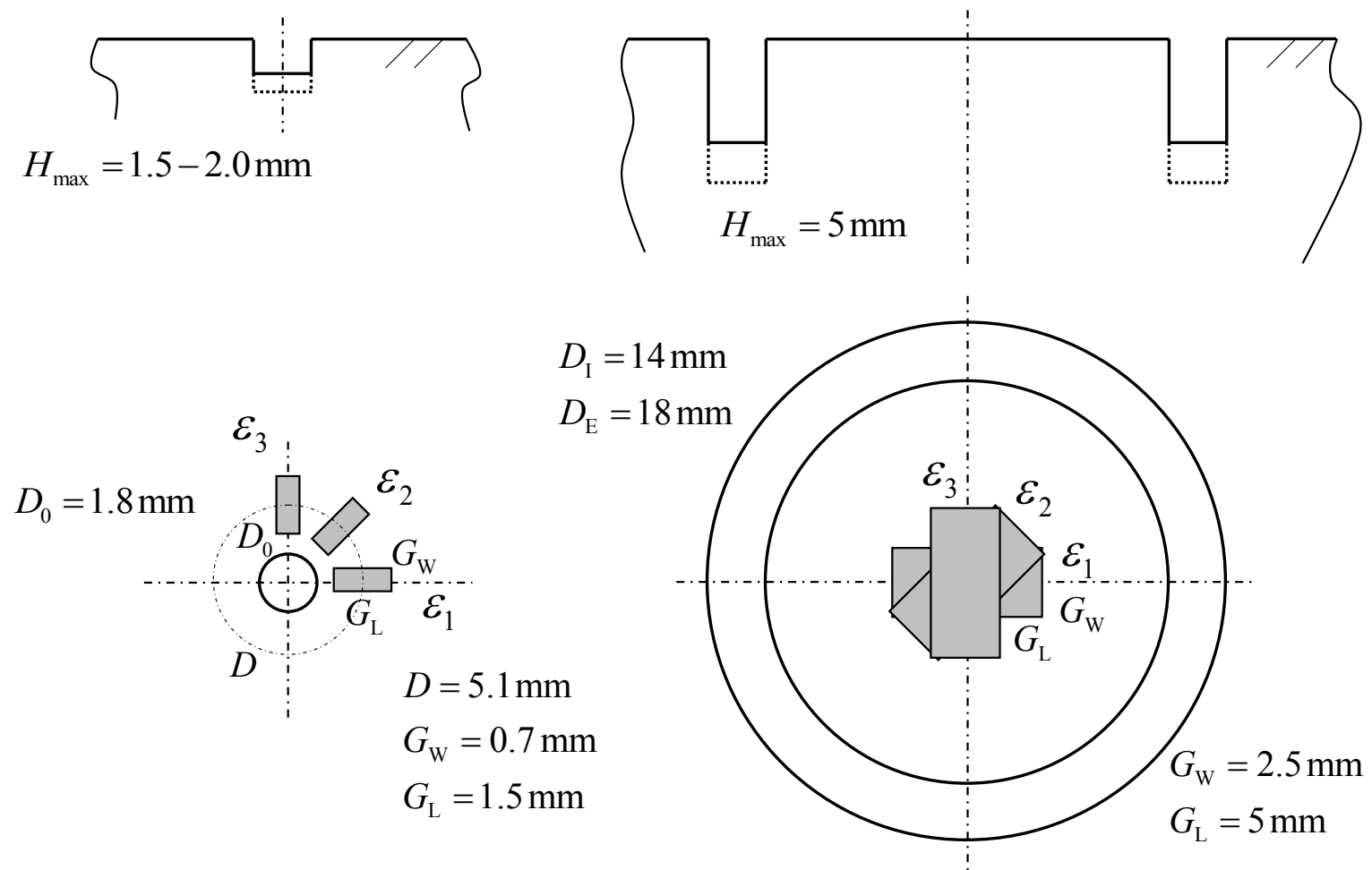

Figure 1. Hole-drilling and ring-core methods, typical dimensions. 
The calculation of the residual stresses, after incremental depth drilling and relieved strain recording, can be performed with the ASTM standard E837 - 13a [1] that introduces the so called "Integral Method". This numerical technique can also be applied to the ring-core $[12,13]$, since the axial-symmetry of the problem, though other techniques are also available, such as the differential method $[14,15]$. The present paper shows an accurate FE model and integral method coefficients derivation for the ring-core, with diameters and grid dimensions as reported in Fig. 1. These coefficients were derived for different depth stepping and different Poisson's ratios. The integral method procedure and the correctness of the proposed coefficients were then validated with a 3D model, completely independent from the axial-symmetric model that was used for the coefficient derivation. Finally, the Tikhonov regularization, as proposed by the ASTM E837 standard was also applied to the ring-core, for an experimental case, showing the effectiveness of this numerical procedure.

\section{FE model for integral method coefficients}

The ring-core method, similarly to the more traditional hole-drilling method, requires a database of computed strains from which the elasticity coefficients can be obtained. Given the linearity of the problem, the generic state of tension can be represented by a basis of solutions, whose terms are simple stress states. The choice of the basis is not unique, however stepped uniform stress distribution is simple, effective and then usual. The state of tension is defined by three components: normal stresses in two orthogonal directions and a component of shear stress. Typically the principal stresses are alternatively used, but in the present problem is preferable to refer to an equibiaxial tension and a pure shear. In the FE model these two components are the loads applied to obtain the database solutions, Fig. 2.
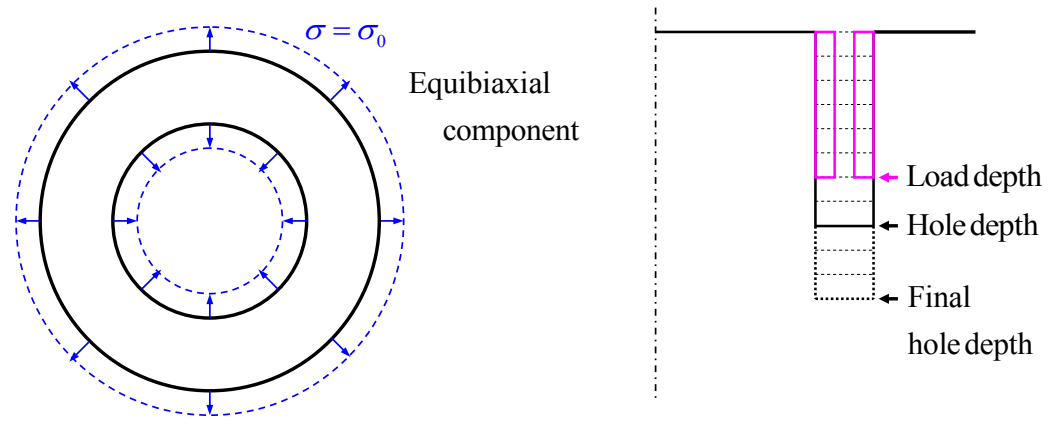

Pure shear component

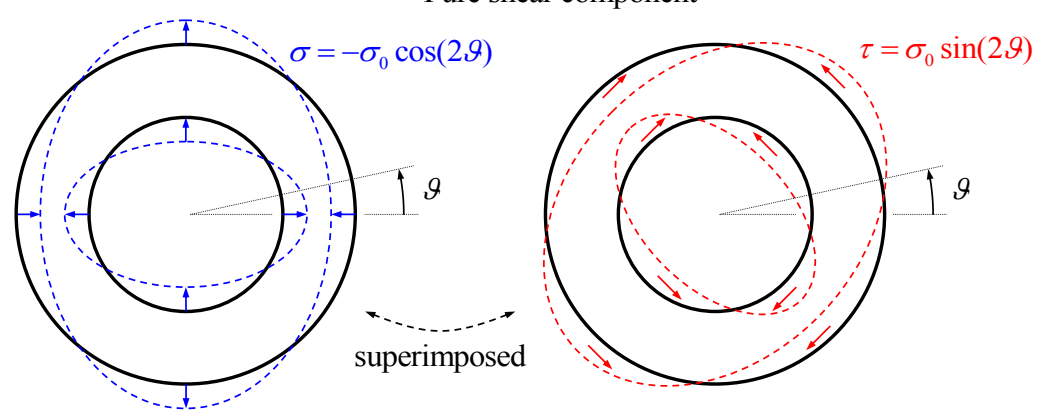

Figure 2. Basis loading conditions to be applied to the FE model.

From a geometrical point of view, the ring-core problem, similarly to the hole-drilling, is axisymmetric, while the load condition is generic (except in special cases). Given the axi-symmetric geometry and the linearity of the problem, the FE plane harmonic can be used (ANSYS Plane 25 element type) in which the model is restricted to the section, Fig. 3, with out of plane load and harmonic angular dependency. The equi-biaxial stress can be modeled with axi-symmetric harmonic order 0 (no angular dependency), while the pure shear stress is the result of the superposition of two components both with harmonic order $2(\cos (2 \vartheta)$ dependency), Fig. 2 . The resolution of the model 
has been set equal to $0.1 \mathrm{~mm}$, so the load was applied on a discrete number of elements, and then the total number of simulations was $2 \times(50 \times(50+1) / 2)=2550$. In this way it was possible to simulate a drilling step of $0.1 \mathrm{~mm}$, or even multiples such as $0.2 \mathrm{~mm}, 0.5 \mathrm{~mm}$, etc. As usual for this kind of simulations, the model was residual stress free, with the groove material already removed and the load applied to both the internal cylindrical surfaces of the groove. Actually, the physical problem is the opposite. Residual stresses are pre-existing, so the material removal relaxes to zero the stresses at the internal surfaces of the groove. Obviously, this need to be taken into account for data processing. It suffices to introduce a minus sign in the relation between stresses and strains.

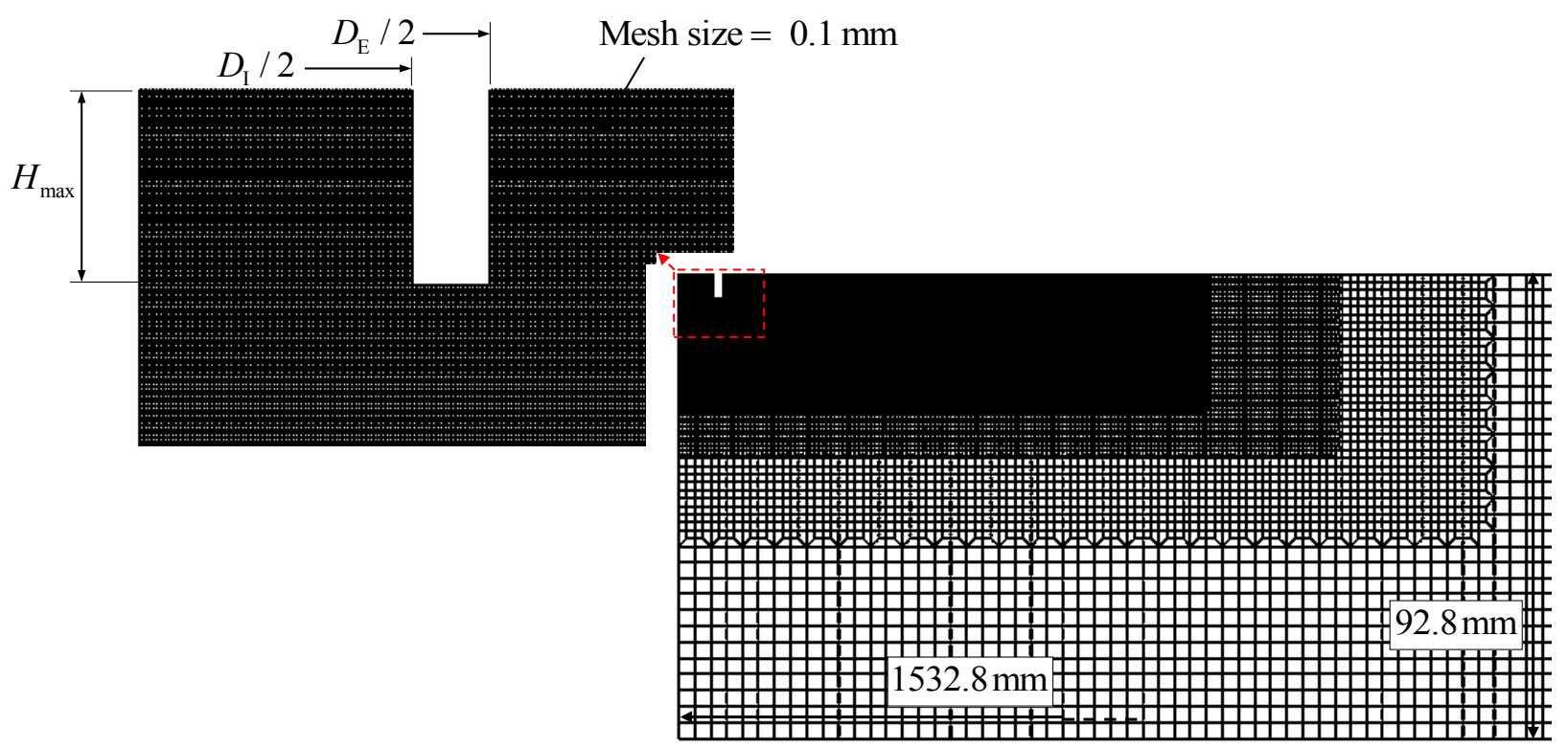

Figure 3. FE plane axi-symmetric harmonic model.

Grid deformations from displacement field. The simulated average strain measured by the grids can be evaluated just by computing the displacement (along each grid direction) at the edge segments, and divide the displacement differences by the grid length, without retrieving any displacement information at intermediate positions of the grids. The whole procedure is detailed summarized below:

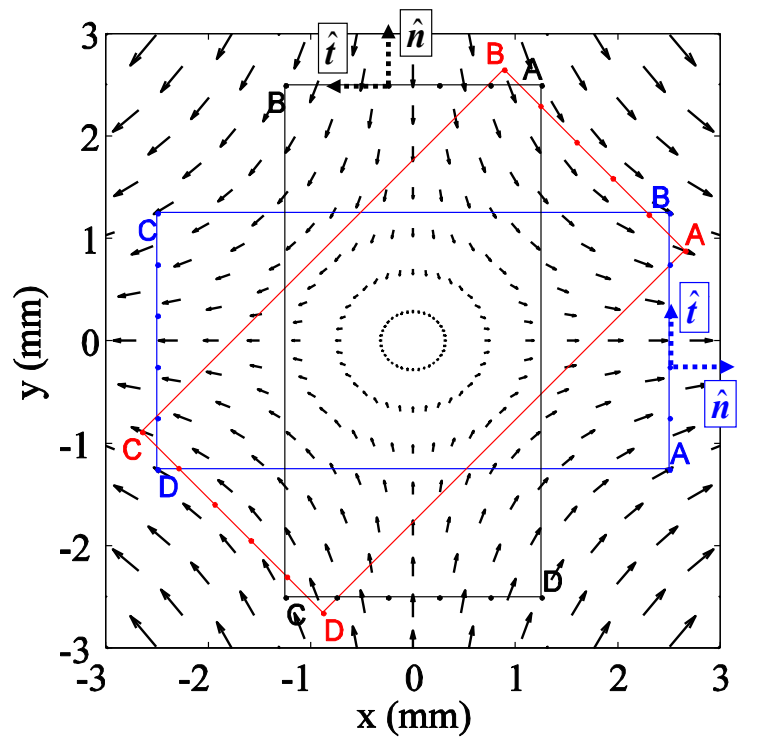

Figure 4. Superposition of strain-gauges to the deformation field.
(1) Set parameters according to the FE model: load, groove diameters and grid geometry.

(2) Define the vertices $A, B, C, D$ of each strain gauge and a large enough number of integration points on sides $A B$ and $C D$ both in cartesian and polar coordinates, Fig. 4.

(3) Introduce the components of the unit vectors transverse $\hat{\boldsymbol{t}}$ and normal $\hat{\boldsymbol{n}}$ (external) to the segments $A B$ and $C D$, at each integration point.

(4) Calculate the displacements along the $x$ and $y$ directions by linear interpolation, at each integration point on sides $A B$ and $C D$.

(5) Deduce the displacement components along the transverse and normal directions at the integration points.

(6) Being the grid only sensitive to the normal direction (transverse sensitivity is very small,

though not zero) the displacements along $\hat{\boldsymbol{t}}$, at integration points, were only considered. Otherwise transverse displacements would be taken into account on lateral sides $B C$ and $D A$. 
(7) Numerically integrate, on the active sides $A B$ and $C D$, by averaging the integration point normal displacements.

(8) Compute the difference between $\mathrm{AB}$ and $\mathrm{CD}$ integrations and divide by the grid length to obtain the average strain.

After the calculation of the simulated strains, the vectors $\boldsymbol{p}$ and $\boldsymbol{q}$, as defined according to ASTM standard [1], were obtained for stepped loads as the grid strains difference between the load at a certain depth and the load to the previous depth. This calculation was performed for $0.1 \mathrm{~mm}$ depth step and also for $0.2 \mathrm{~mm}$ and other multiples. The relation between stresses and strains, as reported in the ASTM standard [1] were then inverted and finally the values $a_{i j}, b_{i j}$ obtained. These coefficients are listed in Tab. 1 , for $1 \mathrm{~mm}$ depth step, and Poisson's ratio $v=0.3$. Other coefficients, for different step sizes and Poisson's ratios were derived but not reported here for brevity.

Table 1. Calibration coefficients for depth step $1 \mathrm{~mm}, v=0.3$, groove and grid dimensions as reported in Fig. 1.

Calibration coefficients matrix $\boldsymbol{a}$

depth, mm

\begin{tabular}{|r|r|r|r|r|r|}
\hline 1.0 & 0.1145 & 0 & 0 & 0 & 0 \\
\hline 2.0 & 0.1966 & 0.1139 & 0 & 0 & 0 \\
\hline 3.0 & 0.2362 & 0.1658 & 0.0815 & 0 & 0 \\
\hline 4.0 & 0.2523 & 0.1847 & 0.1114 & 0.0451 & 0 \\
\hline 5.0 & 0.2575 & 0.1908 & 0.1197 & 0.0598 & 0.0176 \\
\hline
\end{tabular}

Calibration coefficients matrix $\boldsymbol{b}$

depth, mm
\begin{tabular}{|r|r|r|r|r|r|}
\hline 1.0 & 0.1382 & 0 & 0 & 0 & 0 \\
\hline 2.0 & 0.2668 & 0.1529 & 0 & 0 & 0 \\
\hline 3.0 & 0.3710 & 0.2519 & 0.1279 & 0 & 0 \\
\hline 4.0 & 0.4462 & 0.3159 & 0.1952 & 0.0872 & 0 \\
\hline 5.0 & 0.4945 & 0.3559 & 0.2317 & 0.1286 & 0.0487 \\
\hline
\end{tabular}

\section{Validation of calibration coefficients}

A 3D model was used to validate the procedure, Fig. 5 (a). Though coarser as discretization, this model is more simple in terms of applied load and boundary conditions and grid simulated strain calculations. Thus a successful comparison for a generic residual stress, between this 3D model and the results of the procedure shown above, allowed confidence about coefficients correctness and proper application of the integral method. After the 3D model solution, the grid measurements were simulated. The relieved strain were obtained as difference from the applied load condition and the strains generated by the (uniform) residual stress field, easily computed with the Hooke's law, or even calculated with the same FE model without any material removal. Then the integral method, with the coefficients reported in Tab. 1, was implemented and the calculated stresses were finally compared to the imposes stresses. A successful comparison is evident from Fig. 5 (b).

(a)

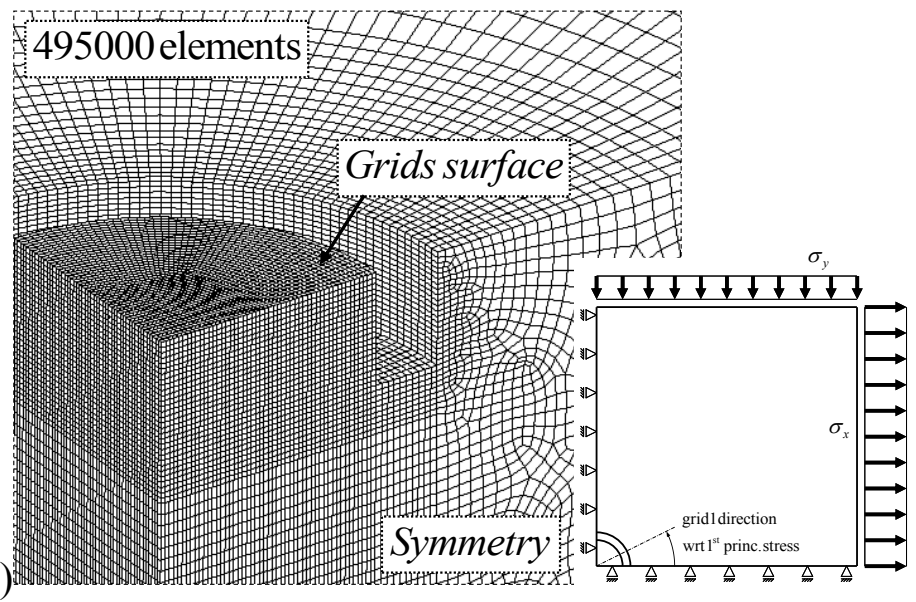

(b)

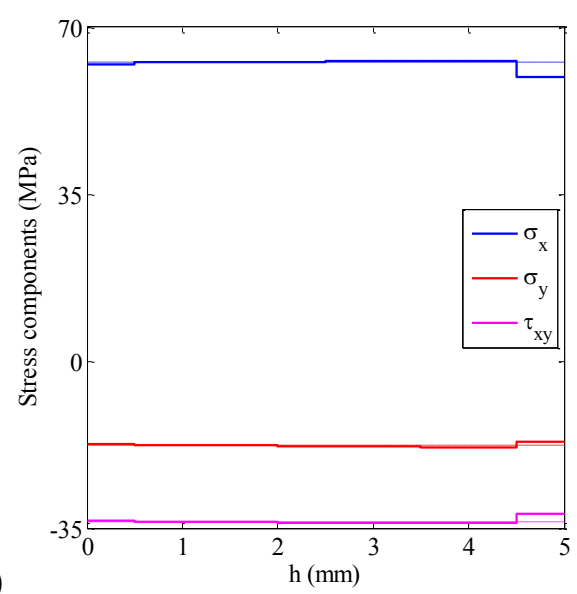

Figure 5. (a) FE 3D model for validation, (b) comparison: imposed vs. back-calculated stresses. 


\section{Tikhonov regularization}

Spurious oscillations caused by the unavoidable experimentally uncertainties, on the measured strains, can be smoothened with different techniques. More specifically, the E837 [1] standard introduced the Tikhonov regularization, Eq. 1, where $c$ is the tri-diagonal "second derivative" matrix and $\alpha_{\mathrm{P}}, \alpha_{\mathrm{Q}}, \alpha_{\mathrm{T}}$ are the regularization factors, for each stress (coupled) component. Obviously, if these factors are set to zero, Eq. 1 just reduces to the unsmoothed integral method.

$$
\begin{aligned}
& \boldsymbol{P}=\frac{E}{1+v}\left(\boldsymbol{a}^{\mathrm{T}} \boldsymbol{a}+\alpha_{\mathrm{P}} \boldsymbol{c}^{\mathrm{T}} \boldsymbol{c}\right)^{-1} \boldsymbol{a}^{\mathrm{T}} \boldsymbol{p} \\
& \boldsymbol{Q}=E\left(\boldsymbol{b}^{\mathrm{T}} \boldsymbol{b}+\alpha_{\mathrm{Q}} \boldsymbol{c}^{\mathrm{T}} \boldsymbol{c}\right)^{-1} \boldsymbol{b}^{\mathrm{T}} \boldsymbol{q} \\
& \boldsymbol{T}=E\left(\boldsymbol{b}^{\mathrm{T}} \boldsymbol{b}+\alpha_{\mathrm{T}} \boldsymbol{c}^{\mathrm{T}} \boldsymbol{c}\right)^{-1} \boldsymbol{b}^{\mathrm{T}} \boldsymbol{t}
\end{aligned}
$$

An example is provided hereafter. The ring-core was applied on a steel component and the integral method implemented. Fig. 6 shows the comparison for the intermediate (and typical) step resolution: $0.5 \mathrm{~mm}$, and it is evident the effect of the regularization. The factor search was performed with the procedure suggested in the E837 standard, and convergence was reached at iteration steps $21,5,11$ for $\boldsymbol{P}, \boldsymbol{Q}, \boldsymbol{T}$ stress components respectively, and the obtained regularization factors were: $\alpha_{\mathrm{P}}=2.131 \times 10^{-4}, \alpha_{\mathrm{Q}}=2.233 \times 10^{-3}, \alpha_{\mathrm{T}}=1.800 \times 10^{-2}$.

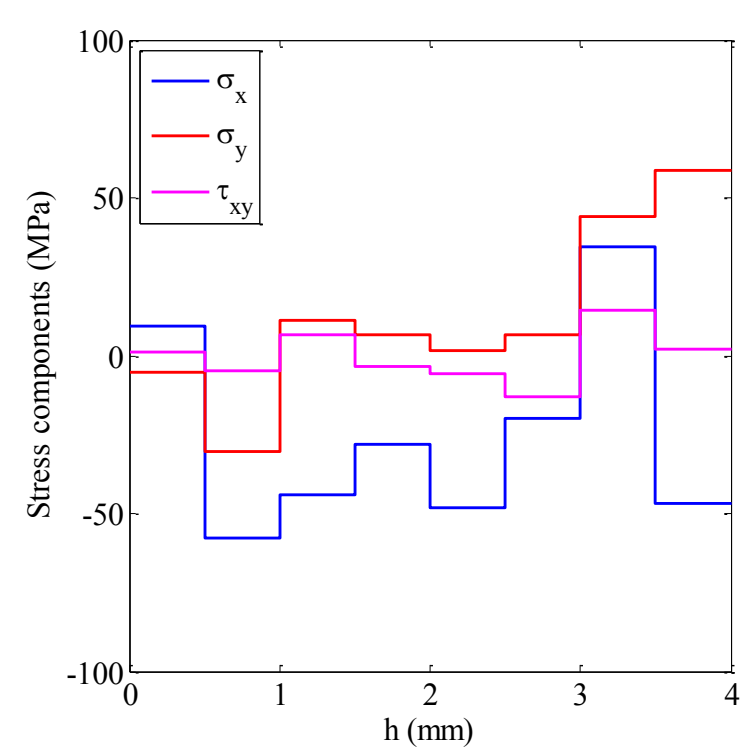

(a)

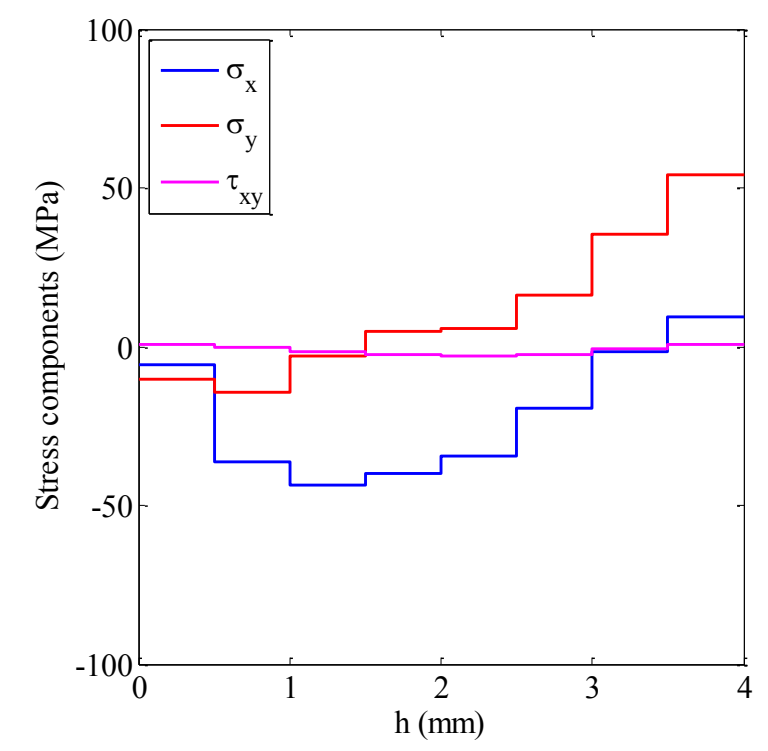

(b)

Figure 6. Components of residual stresses: (a) integral method, (b) Tikhonov regularization.

\section{Conclusions}

This paper shows the application of the integral method to the ring-core technique for measuring the residual stress distribution up to (relatively) higher depths. A harmonic axi-symmetric finite element model was described along with the procedure to numerically deduce the grid average strains and then the calibration matrices were obtained and reported. Validation of the procedure was also provided, by means of a 3D FE model. Finally, an application with experimental data was used to show the performance of the Tikhonov regularization, as proposed in the ASTM standard for the hole-drilling method, with the here derived ring-core coefficients. 


\section{Acknowledgement}

Regione Toscana is acknowledged for funding this project: Bando 2009. Reg. (CE) n. 1083/2006. POR CReO/FERS 2007-2013. Linea di intervento $1.3 \mathrm{a}$.

\section{References}

[1] ASTM E837 - 13a. Standard Test Method for Determining Residual Stresses by the HoleDrilling Strain-Gage Method. (2013).

[2] M. Beghini, L. Bertini, C. Santus. A procedure for evaluating high residual stresses using the blind hole drilling method, including the effect of plasticity. Journal of Strain Analysis for Engineering Design 45 (2010), 301-318.

[3] M. Beghini, C. Santus, E. Valentini, A. Benincasa. Experimental verification of the hole drilling plasticity effect correction. Materials Science Forum 681 (2011), 151-158.

[4] E. Valentini, C. Santus, M. Bandini. Residual stress analysis of shot-peened aluminum alloy by fine increment hole-drilling and X-ray diffraction methods. Procedia Engineering 10 (2011), 3582-3587.

[5] E. Valentini, M. Beghini, L. Bertini, C. Santus, M. Benedetti. Procedure to perform a validated incremental hole drilling measurement: Application to shot peening residual stresses. Strain 47 (2011), Suppl. 1, e605-e618.

[6] E. Valentini, A. Benincasa, C. Santus. Bending test rig for validating the hole drilling method residual stress measurement. Materials Science Forum 768-769 (2014), 150-157.

[7] E. Procter, E.M. Beaney. Trepan or Ring Core Method, Centre-Hole Method, Sach's Method, Blind Hole Methods, Deep Hole Technique. Adv in Sur Treat, Technol - Appl - Eff. Residual Stresses. 4 (1987) 165-198.

[8] W. Böhm, E. Stücker, H. Wolf. Principles and potential applications of the ring-core method for determining residual stresses. RAM. 4 (1988) 5-10.

[9] J. Václavík, O. Weinberg, P. Bohdan, J. Jankovec, S. Holý. Evaluation of Residual Stresses using Ring Core Method. EPJ Web of Conferences 6, 44004 (2010) p1-p6.

[10] S. Keil. On-line evaluation of measurement results during the determination of residual stress using strain gages. RAM. 9 (1995) 15-20.

[11]E. Valentini, A. Benincasa, L. Bertelli. Un sistema automatico per la misura delle tensioni residue con il metodo della cava anulare. $40^{\circ}$ convegno AIAS. (2011).

[12]A. Ajovalasit, G. Petrucci, B. Zuccarello. Determination of Nonuniform Residual Stresses Using the Ring-Core Method. Journal of Engineering Materials and Technology, Transactions of the ASME 118 (1996) 224-228

[13]B. Zuccarello. Optimization of depth increment distribution in the ring-core method. Journal of Strain Analysis for Engineering Design. 31 (1996) 251-258.

[14]A. Civín, M. Vlk. Ring-Core Residual Stress Measurement: Analysis of Calibration Coefficients for Incremental Strain Method. Bulletin of Applied Mechanics 6 (2010), 77-83.

[15]A. Civín, M. Vlk. Determination of principal residual stresses' directions by incremental strain method. Applied and Computational Mechanics 5 (2011) 5-14. 